\title{
Assessment of HER2 testing patterns, HER2+ disease, and the utilization of HER2-directed therapy in early breast cancer
}

This article was published in the following Dove Press journal:

Breast Cancer: Targets and Therapy

29 October 2014

Number of times this article has been viewed

\author{
David D Stenehjem ${ }^{1,2}$ \\ Minkyoung Yoo' \\ Sudhir K Unni' \\ Mukul Singhal' \\ Hillevi Bauer ${ }^{\prime}$ \\ Kim Saverno' \\ Cheng Quah ${ }^{3}$ \\ Anthony Masaquel ${ }^{3}$ \\ Diana I Brixner ${ }^{1,4}$ \\ 'Pharmacotherapy Outcomes \\ Research Center (PORC), College \\ of Pharmacy, University of Utah, \\ Salt Lake City, UT, USA; ${ }^{2}$ Huntsman \\ Cancer Institute, Salt Lake City, UT, \\ USA; ${ }^{3}$ Genentech, Inc., South San \\ Francisco, CA, USA; ${ }^{4}$ Program in \\ Personalized Health Care, University \\ of Utah, Salt Lake City, UT, USA
}

Correspondence: David D Stenehjem Pharmacotherapy Outcomes Research Center, 30 South 2000 East, Room 4834 (4th Floor),

Salt Lake City, UT 84I I2, USA

Tel +l 80I 5879715

Email david.stenehjem@hsc.utah.edu
Context: Determining human epidermal growth factor receptor 2 (HER2) status is critical for the management of early-stage breast cancer (ESBC). An understanding of HER2 testing practices can provide insight into how test results influence the use of HER2-directed therapy.

Objective: To assess HER2 testing, HER2+ disease, and HER2-directed therapy in ESBC at the Huntsman Cancer Institute before and after the 2007 American Society of Clinical Oncology and College of American Pathologist (ASCO/CAP) guidelines on HER2 testing were published.

Methods: Patients were identified from an institutional tumor registry. HER2 testing patterns and results were examined using a chart review of pathology and clinical notes. Patient characteristics, HER2+ rate, and trastuzumab use were evaluated descriptively. Discordance rate with reflex testing (immunohistochemistry $[\mathrm{IHC}] 2+$ retested by fluorescence in situ hybridization [FISH]) was also evaluated.

Results: A total of 1,459 women were included (mean age: 57 years). The rate of HER $2+$ disease was $17 \%$ (number $[\mathrm{N}]=245$ ). The discordance rate between $\mathrm{IHC} 2+$ and FISH was $10 \%$. After the 2007 ASCO/CAP guidelines, fewer tumors were classified as IHC3+ (16\% post- versus $21.9 \%$ pre-2007), more tumors were characterized as IHC $2+(26.4 \%$ post- versus $20.7 \%$ pre-2007), and the overall HER $2+$ rate was decreased $(18.7 \%$ versus $21.9 \%)$, but this was not statistically significant $(P=0.519)$. Most patients with HER2+ ESBC received HER2-targeted therapy $(\mathrm{N}=185)$.

Conclusion: The HER $2+$ rate was $17 \%$ and within the range of the reported rates in the literature. Reflex testing identified additional HER2+ tumors by approximately $10 \%$, and should be considered a potential quality indicator. ASCO/CAP HER2 testing guidelines in 2007 appeared to impact the interpretation and classification of HER2+ tumors.

Keywords: human epidermal growth factor receptor 2, HER2, early-stage breast cancer, immunohistochemistry, FISH, HER2-targeted therapy

\section{Introduction}

Assessment of the expression of human epidermal growth factor receptor 2 (HER2) is recommended by the joint American Society of Clinical Oncology and the College of American Pathologists (ASCO/CAP) guidelines and the National Comprehensive Cancer Network (NCCN) in all patients with invasive breast cancer. ${ }^{1-3}$ Abnormal HER2 amplification is present in $10 \%-34 \%$ of invasive breast cancer tumors. ${ }^{4}$ HER2-amplified (HER2+) tumors were historically associated with poor prognosis; however, HER2directed therapies (trastuzumab, lapatinib, pertuzumab, and ado-trastuzumab emtansine) have demonstrated improved outcomes. A recently published meta-analysis of randomized trials investigating the use of trastuzumab (Genentech, Inc., San Francisco, CA, USA), a HER2 receptor antagonist, among women with HER2+ early-stage breast cancer 
(ESBC) found that patients receiving treatment regimens that included trastuzumab had significantly improved survival (all $P<0.0001$ ). ${ }^{5,6}$ This emphasizes the importance of testing for HER2 positivity to provide appropriate therapy for patients with HER2+ breast cancer.

Determining HER2 biomarker expression in ESBC and subsequent prescribing patterns can be instrumental for understanding how these agents are used in routine clinical practice to improve outcomes. Several tests are approved by the United States Food and Drug Administration (FDA) to determine HER2 status, including immunohistochemistry (IHC), fluorescence in situ hybridization (FISH), and chromogenic in situ hybridization. ${ }^{7}$ The most frequently utilized tests are IHC and FISH, of which IHC is used in $80 \%$ of tested tumors, ${ }^{4}$ but this methodology can have a discordance rate up to $20 \%$ when followed by FISH testing in a small laboratory. ${ }^{8}$

In 2007, ASCO/CAP estimated that approximately $20 \%$ of tests may have been incorrect, and guidelines were released standardizing the HER2 testing methodology with clear definitions for HER2 positive, negative, and equivocal tumors by IHC and FISH. ${ }^{2}$ Recently, these guidelines were updated (2013), readdressing the methodology that defines the HER2equivocal and positive groups by IHC and FISH, and which includes new testing platforms. ${ }^{9,10}$ The 2007 guidelines were reverted by recent guidelines, with the recommendation for circumferential, intense, and complete membrane staining in $>30 \%$ of cells for IHC equivocal (IHC2+) and positive (IHC3+) tumors to the previous definition of staining in $>10 \%$ of cells, which was used prior to 2007 and was included in the adjuvant trastuzumab trials. ${ }^{10}$

The purpose of this study was to assess HER2 testing practices, the rate of HER2+ disease, and trastuzumab use in patients diagnosed with ESBC. Our study period allowed us to compare HER2 testing patterns and outcomes prior to and after the implementation of the 2007 ASCO/CAP guidelines on HER2 testing.

\section{Methods}

\section{Study population}

This study is an observational study consisting of a cohort of female patients diagnosed with ESBC from January 1, 2005 through December 31, 2012. Patients were identified in the Huntsman Cancer Institute Tumor Registry (HCI-TR), a Surveillance, Epidemiology, and End Results (SEER) reporting registry in Utah (USA). The HCI-TR is linked to the enterprise data warehouse for the University of Utah Hospital containing a comprehensive electronic health record (EHR) for all patients since 1995. Patients were included based on age ( $\geq 18$ years); documented stage 1 through $3 \mathrm{~A}$ breast cancer by the International Classification of Diseases (ICD) for Oncology site, as well as histology codes in the HCI-TR; and patients were required to have $\geq 2$ visits separated by $\geq 30$ days with ICD-9 codes for breast cancer (174.x) between January 1, 2005 and April 1, 2013. Patients with secondary neoplasms (ICD-9-CM 196.x-198.x) were excluded from the study if identified within \pm 90 days of diagnosis. Patients were followed until April 1, 2013 or death, whichever occurred earlier. The index date was defined as the date of diagnosis of breast cancer in the HCI-TR. Patient data \pm 90 days from the index date were collected to evaluate clinical characteristics at diagnosis. A waiver of informed consent was granted, and institutional review board approval was obtained from the University of Utah.

\section{Study outcomes}

HER2 testing was determined by a chart review of pathology reports and physician notes contained in the EHR from 90 days prior to the index date to April 1, 2013. HER2 testing at the HCI was conducted by ARUP Laboratories, a national reference laboratory and a CAP-accredited laboratory affiliated with the University of Utah. The US FDA-approved HercepTest ${ }^{\mathrm{TM}}$ (Dako Denmark A/S, Glostrup, Denmark) or PATHWAY HER-2/neu (4B5) kit (Ventana Medical Systems, Inc., Tucson, AZ, USA) were used for IHC testing, and the US FDA-approved Vysis PathVysionTM HER2 DNA Probe kit (Abbott Laboratories, Abbott Park, IL, USA) was used for FISH testing according to the manufacturer's instructions during the study period.

The type of test performed for each patient (FISH, IHC, or other) and the test results were recorded. All HER2 test results from the patient's medical chart (pathology reports) were considered when making a final determination of the patient's HER2 status. When the results were ambiguous or mixed, the final HER2 status was determined based upon the oncologist-documented consensus of the patient's HER2 status, which was also cross-referenced with the tumor registry, when available.

The IHC and FISH results were used to categorize patients' tumors as HER2+, HER2-, or equivocal based on the 2007 ASCO/CAP guidelines ${ }^{11}$ at the time of testing. Reflexive testing by FISH for an $\mathrm{IHC} 2+$ result was assessed within 30 days of an IHC2+ result, according to the ASCO/ CAP 2007 clinical guidelines. The rate of concordance and discordance between the reflex testing results was analyzed. For this study, the number of ESBC patients receiving HER2directed therapies, irrespective of their HER2 status, was 
captured from inpatient and outpatient records, institutional retail pharmacies, electronic prescription orders, and by chart review. Lastly, HER2 testing results were assessed before (2005-2006) and after (2007-2012) publication of the ASCO/ CAP guidelines in 2007 .

\section{Statistical analysis}

Descriptive statistics including means and standard deviations (SDs) for continuous variables, as well as counts and percentages for categorical variables were utilized to summarize the findings. The chi-square test of homogeneity was used to determine whether the distribution of the HER2 test results was similar across clinical characteristics. The distribution of HER2 test results was also compared by patient characteristics such as age, race/ethnicity, insurance plan type, Utah residency, as well as by clinical characteristics, such as year of diagnosis, estrogen receptor (ER) and progesterone receptor (PR) status, lymph node involvement, histologic grade, American Joint Committee on Cancer Tumor Node Metastasis (TNM) staging, and modified Charlson Comorbidity Index. ${ }^{12}$ All statistical tests were evaluated at an $\alpha=0.05$ level.

\section{Results}

A total of 2,389 female patients with breast cancer were identified between 2005 and 2012 (Figure 1). The overall cohort size for this analysis was 1,458 patients with stage 1 (49\%; number $[\mathrm{n}]=710), 2 \mathrm{~A}(26 \% ; \mathrm{n}=374), 2 \mathrm{~B}(13 \% ; \mathrm{n}=197)$; and $3 \mathrm{~A}(12 \%, \mathrm{n}=168)$ after applying the inclusion and exclusion criteria. Of these 1,458 female patients with ESBC, 26 patients ( $2 \%$ ) had an unknown HER2 status; neither chart review nor the tumor registry contained any information on the patients' HER2 status. A total of 1,233 patients (86\%) had detailed information in the EHR regarding HER2 testing history, and 199 patients only had their final HER2 status documented without a HER2 testing history.

The average (SD) age of the women at the time of ESBC diagnosis was $57( \pm 13)$ years (Table 1$)$. The majority were white $(84.2 \%)$, from the state of Utah $(77.4 \%)$, and had commercial medical insurance coverage (51.0\%). Modified Charlson Comorbidity scores were evenly distributed with scores of $0,1,2$, and $\geq 3$ corresponding to $26.5 \%, 26.1 \%$, $20.6 \%$, and $26.8 \%$ of all ESBC patients, respectively. Tumor grade differed according to tumor stage at diagnosis. More than half $(54.2 \%)$ of those diagnosed with stage $3 \mathrm{~A}$ disease had grade 3 tumors. Approximately one-fourth (25.3\%) of patients with stage 1 disease had grade 3 tumors.

A total of 1,802 tests for HER2+ status were performed in the entire cohort $(n=1,458)$. Of all tests conducted, IHC was the most commonly ordered (75.4\%), followed by FISH (23.5\%), and other test types (1.1\%). IHC was also most often to be the first test conducted (96.8\%), followed by FISH $(2.9 \%)$, and other tests $(0.3 \%)$. A total of 245 tumors $(17 \%)$ were determined to be HER2+. Figure 2 presents the final HER2 status stratified by age, estrogen receptor and progesterone receptor

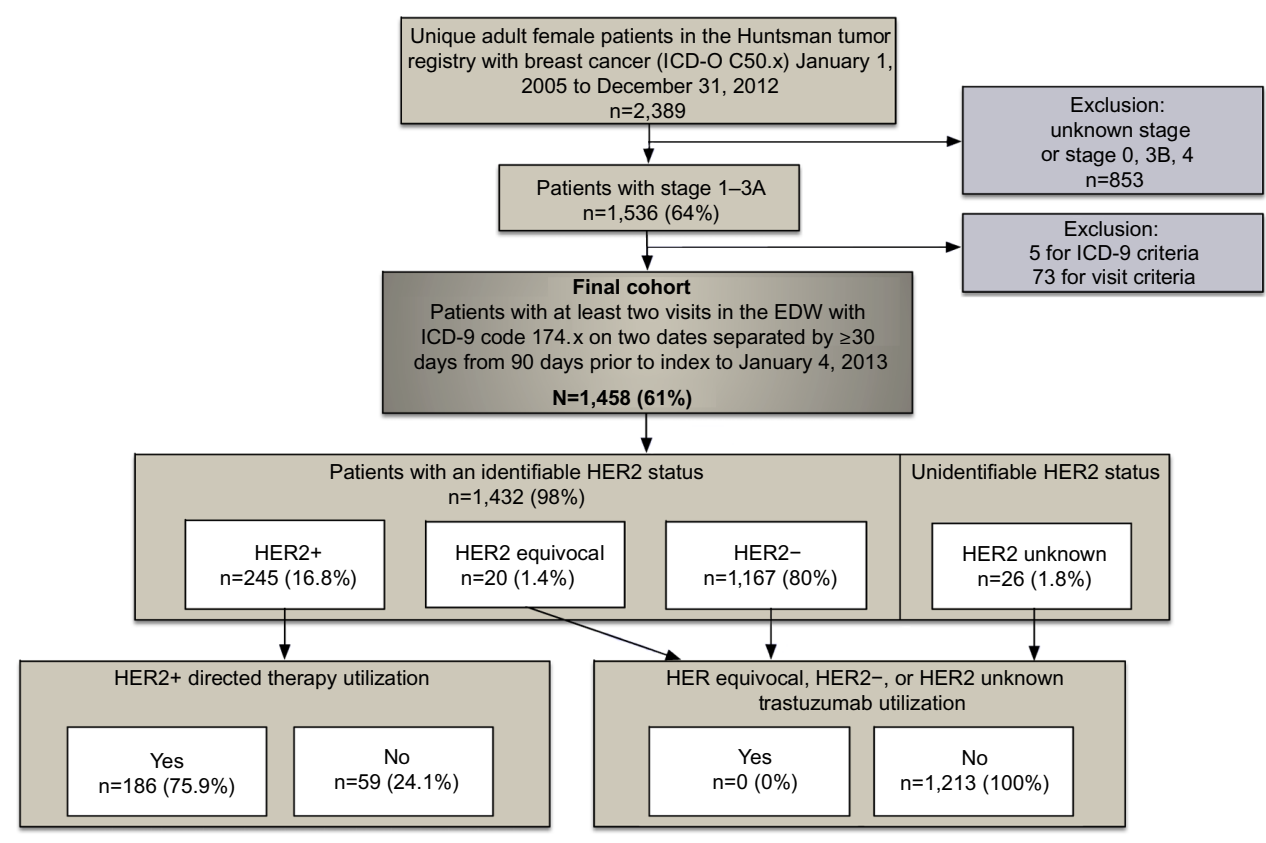

Figure I Patient flow, HER2 status, and HER2-directed therapy utilization.

Abbreviations: ICD-O, International Classification of Diseases for Oncology; n, number; ICD, International Classification of Diseases; EDW, enterprise data warehouse; HER2, human epidermal growth factor receptor 2 . 
Table I Demographic and clinical characteristics of the study population stratified based on stage $(\mathrm{N}=\mathrm{I}, 458)$

\begin{tabular}{|c|c|c|c|c|c|}
\hline & $\begin{array}{l}\text { Overall } \\
(\mathrm{N}=1,458) \\
\end{array}$ & $\begin{array}{l}\text { Stage I } \\
(\mathrm{N}=7 \text { I9) } \\
\end{array}$ & $\begin{array}{l}\text { Stage 2A } \\
(\mathrm{N}=374)\end{array}$ & $\begin{array}{l}\text { Stage 2B } \\
(\mathrm{N}=197) \\
\end{array}$ & $\begin{array}{l}\text { Stage 3A } \\
(\mathrm{N}=168) \\
\end{array}$ \\
\hline & n (\%) & n (\%) & n (\%) & n (\%) & n (\%) \\
\hline Age (mean, SD) & $(57,13)$ & $(59,13)$ & $(55,13)$ & $(56,13)$ & $(53,13)$ \\
\hline $18-30$ years & $30(2.1)$ & $9(1.3)$ & $12(3.2)$ & $3(1.5)$ & $6(3.6)$ \\
\hline $31-45$ years & $258(17.7)$ & $92(12.8)$ & 74 (19.8) & $43(21.8)$ & $49(29.2)$ \\
\hline 46-64 years & $731(50.1)$ & $356(49.5)$ & $200(53.5)$ & $100(50.8)$ & $75(44.6)$ \\
\hline $65-79$ years & $361(24.8)$ & $213(29.6)$ & $72(19.3)$ & $43(21.8)$ & $33(19.6)$ \\
\hline$\geq 80$ years & $78(5.4)$ & $49(6.8)$ & $16(4.3)$ & $8(4.1)$ & $5(3)$ \\
\hline \multicolumn{6}{|l|}{ Race } \\
\hline Caucasian/white & I,228 (84.2) & $608(84.6)$ & $318(85)$ & I 70 (86.3) & I 32 (78.6) \\
\hline African-American & $10(0.7)$ & $2(0.3)$ & $5(1.3)$ & $2(I)$ & I (0.6) \\
\hline Asian & $29(2)$ & $16(2.2)$ & $4(1.1)$ & $5(2.5)$ & $4(2.4)$ \\
\hline Other & $78(5.4)$ & $30(4.2)$ & $24(6.4)$ & $12(6.1)$ & $12(7.1)$ \\
\hline Unknown & $113(7.7)$ & $63(8.8)$ & $23(6.2)$ & $8(4.1)$ & $19(11.3)$ \\
\hline \multicolumn{6}{|l|}{ Plan type } \\
\hline Commercial & $792(54.3)$ & $367(5 \mathrm{I})$ & $213(57)$ & $116(58.9)$ & $96(57.1)$ \\
\hline Medicare & $468(32.1)$ & $268(37.3)$ & $104(27.8)$ & $52(26.4)$ & $44(26.2)$ \\
\hline Medicaid & $60(4.1)$ & $21(2.9)$ & $16(4.3)$ & $10(5.1)$ & $13(7.7)$ \\
\hline Other/unknown & $138(9.5)$ & $63(8.8)$ & $4 I(I I)$ & $19(9.6)$ & 15 (8.9) \\
\hline \multicolumn{6}{|l|}{ Region } \\
\hline Utah & I, I 29 (77.4) & 553 (76.9) & $298(79.7)$ & I 58 (80.2) & $120(7 \mid .4)$ \\
\hline Non-Utah & $329(22.6)$ & $166(23.1)$ & $76(20.3)$ & $39(19.8)$ & $48(28.6)$ \\
\hline \multicolumn{6}{|l|}{ Estrogen receptor status } \\
\hline Negative & $286(19.6)$ & $107(14.9)$ & $87(23.3)$ & $4 \mathrm{I}(20.8)$ & $51(30.3)$ \\
\hline Equivocal & $5(0.4)$ & $3(0.4)$ & $0(0.0)$ & I $(0.5)$ & I (0.6) \\
\hline Positive & I, I 49 (78.8) & $599(83.3)$ & $282(75.4)$ & $153(77.7)$ & $115(68.5)$ \\
\hline Unknown & $18(1.2)$ & $10(1.4)$ & $5(1.3)$ & $2(I)$ & I (0.6) \\
\hline \multicolumn{6}{|l|}{ Progesterone receptor status } \\
\hline Negative & $439(30.1)$ & I $78(24.8)$ & $122(32.6)$ & $64(32.5)$ & $75(44.6)$ \\
\hline Equivocal & $17(1.2)$ & $7(I)$ & $4(I . I)$ & $2(1)$ & $4(2.4)$ \\
\hline Positive & $980(67.2)$ & $523(72.7)$ & $24 \mid(64.4)$ & $128(65)$ & $88(52.4)$ \\
\hline Unknown & $22(1.5)$ & II (I.5) & $7(1.9)$ & $3(1.5)$ & $\mathrm{I}(0.6)$ \\
\hline \multicolumn{6}{|l|}{ Final HER2 status } \\
\hline Negative & I,I67 (80) & $595(82.8)$ & $308(82.4)$ & $142(72.1)$ & $122(72.6)$ \\
\hline Equivocal & $20(1.4)$ & $13(1.8)$ & $6(1.6)$ & $0(0.0)$ & I (0.6) \\
\hline Positive & $245(16.8)$ & $90(12.5)$ & $55(14.7)$ & $55(27.9)$ & $45(26.8)$ \\
\hline Unknown & $26(1.8)$ & $21(2.9)$ & $5(1.3)$ & $0(0.0)$ & $0(0.0)$ \\
\hline \multicolumn{6}{|l|}{ Lymph node status } \\
\hline Evaluated, negative & $847(58.1)$ & $636(88.5)$ & $194(51.9)$ & $14(7.1)$ & $3(1.8)$ \\
\hline Positive & $339(23.2)$ & $0(0.0)$ & $83(22.2)$ & $108(54.8)$ & $148(88.1)$ \\
\hline Not evaluated/unknown & $272(18.7)$ & $83(11.5)$ & $97(25.9)$ & $75(38.1)$ & $17(10.1)$ \\
\hline \multicolumn{6}{|l|}{ Tumor histologic grade } \\
\hline I & $306(2 I)$ & $224(3 \mid .2)$ & $47(12.5)$ & $21(10.7)$ & $14(8.3)$ \\
\hline 2 & $610(41.8)$ & $313(43.5)$ & $145(38.8)$ & $89(45.2)$ & $63(37.5)$ \\
\hline 3 & $542(37.2)$ & $182(25.3)$ & $182(48.7)$ & $87(44.1)$ & 91 (54.2) \\
\hline \multicolumn{6}{|l|}{ TNM staging } \\
\hline TI (tumor $\leq 20 \mathrm{~mm})$ & $886(60.8)$ & $706(98.2)$ & $142(38)$ & $7(3.5)$ & $31(18.4)$ \\
\hline T2 $(20 \mathrm{~mm}<$ tumor $\leq 50 \mathrm{~mm})$ & $464(31.8)$ & $\mathrm{I}(0.1)$ & $225(60.1)$ & $174(88.3)$ & $64(38.1)$ \\
\hline $\mathrm{T} 3$ (tumor $\geq 50 \mathrm{~mm}$ ) & $80(5.5)$ & $0(0)$ & $0(0)$ & $15(7.6)$ & $65(38.7)$ \\
\hline $\begin{array}{l}\text { T4 (tumor of any size with direct extension } \\
\text { to the chest wall and/or to the skin) }\end{array}$ & $0(0)$ & $0(0)$ & $0(0)$ & $0(0)$ & $0(0)$ \\
\hline Unknown/other & $28(1.9)$ & $12(1.7)$ & $7(1.9)$ & $\mathrm{I}(0.6)$ & $8(4.8)$ \\
\hline \multicolumn{6}{|l|}{ Modified Charlson Comorbidity score } \\
\hline 0 & $386(26.5)$ & $147(20.4)$ & $110(29.4)$ & $61(30.9)$ & $68(40.5)$ \\
\hline I & $380(26.1)$ & $186(25.9)$ & $107(28.6)$ & $49(24.9)$ & $38(22.6)$ \\
\hline 2 & $300(20.6)$ & $169(23.5)$ & $70(18.7)$ & $33(16.7)$ & $28(16.7)$ \\
\hline$\geq 3$ & $392(26.8)$ & $217(30.2)$ & $87(23.3)$ & $54(27.5)$ & $34(20.2)$ \\
\hline
\end{tabular}

Abbreviations: $\mathrm{N}$, total number; $\mathrm{n}$, sample number; SD, standard deviation; HER2, human epidermal growth factor receptor 2; TNM, Tumor Node Metastasis. 

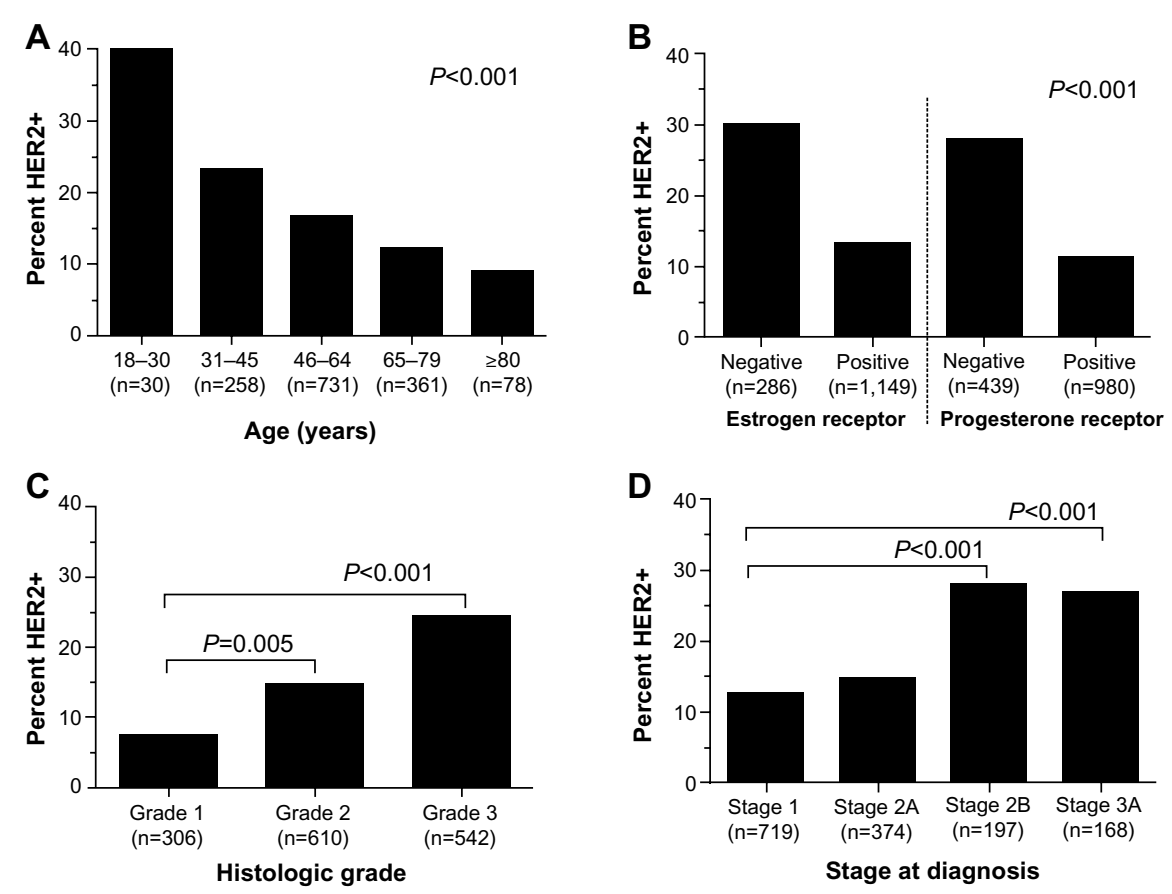

Figure 2 HER2 positivity by (A) age, (B) estrogen receptor/progesterone receptor, (C) grade, and (D) stage. Abbreviations: n, number; HER2, human epidermal growth factor receptor 2.

(ER/PR) expression, tumor grades, and stages of breast cancer. Patients between the ages of 18 years and 30 years had the highest rate of HER2 positivity (40.0\%) followed by those aged $31-45$ years $(23.3 \%), 46-64$ years $(16.7 \%), 65-79$ years $(12.2 \%)$, and $>80$ years $(9.0 \%)$. The highest percent of HER2+ tumors was found in hormone receptor (HR)-negative tumors $(31.6 \% ; 84$ of 266 patients) compared to HR+ tumors (12.7\%; 146 of 1,148 patients). In terms of proportion, of 245 HER $2+$ tumors, $60 \%$ were HR+, $34 \%$ were HR-, and $6 \%$ were unknown. HER positivity was more frequent in grade 3 tumors $(24.5 \%)$ than grade $1(7.5 \%)$ or $2(14.6 \%)$. Stage also correlated with HER2 positivity (Figure 2).

HER2-directed therapy was administered in 186 of 245 (76\%) HER2+ patients. A total of 185 patients with HER2+ ESBC received adjuvant trastuzumab and one received adjuvant lapatinib on a clinical trial protocol. No HER2- or equivocal patients received HER2-directed therapies (Figure 1). A total of 59 patients did not receive HER2-directed therapy, yet had HER2+ tumors. The most commonly reported reason for not administering trastuzumab was a low risk of recurrence within these patients $(n=18)$. Other documented reasons for the lack of use of HER2-directed therapies in patients with HER2+ tumors included loss to follow-up ( $n=9)$, unknown $(\mathrm{n}=11)$, not clinically appropriate due to age or comorbidity $(n=10)$, patient relocated $(n=4)$, and patient declined $(n=7)$. One-third of patients with HER2+ tumors who did not receive HER2-directed therapies were diagnosed in 2005 and 2006.
Reflex testing identified 29 (10\%) additional HER2+ patients by retesting IHC2+ results with FISH. IHC2+ and FISH-negative results occurred in 240 of 291 tests (82.5\%).

Lastly, segmentation of the HER2 test results before (2005-2006) and after (2007-2012) the publication of the ASCO/CAP HER2 testing guidelines in 2007 is presented in Table 2. Of all HER2 tests conducted, IHC was performed $78.6 \%$ and $75.3 \%$ of the time, and FISH was performed $21.4 \%$ and $24 \%$ of the time before and after the 2007 ASCO/CAP HER2 testing guidelines were published, respectively. IHC test results of 0 or $1+$ were similar between time periods, encompassing $56.6 \%$ versus $56.1 \%$ of IHC test results pre- and post-2007, respectively. However, post-guideline implementation, more tumors were characterized as IHC2+ $(26.4 \%$ versus $20.7 \%$ [postversus pre-2007, respectively]; $P \leq 0.001)$ and fewer tumors were classified as IHC3+ $(16 \%$ versus $21.9 \% ; P<0.001)$, and an increased utilization of reflex testing was also observed between the time periods ( $24 \%$ versus $10 \%)$. FISH test results were similar before and after the 2007 ASCO/CAP HER2 testing guidelines were published, with $13.5 \%$ of the test results considered HER2-amplified after 2007 when compared to $12.1 \%$ prior to 2007 . Although no statistically significant difference in the distribution of the final HER2 status over the two periods was observed $(P=0.519)$, more tumors were classified as HER2- $(79.7 \%$ versus 76.4\% [post- versus pre-2007]) and fewer were 
Table 2 Status of HER2 testing* before and after the establishment of ASCO/CAP guidelines

\begin{tabular}{|c|c|c|c|c|}
\hline \multirow[t]{3}{*}{ Test type } & \multirow{3}{*}{$\begin{array}{l}\text { Overall } \\
\mathrm{n}(\%)\end{array}$} & \multirow{3}{*}{$\begin{array}{l}\text { Before NCCN guidelines } \\
(2005-2006) \\
n(\%)\end{array}$} & \multirow{3}{*}{$\begin{array}{l}\text { After NCCN guidelines } \\
(2007-20 I 2) \\
n(\%)\end{array}$} & \multirow[t]{3}{*}{$P$-value } \\
\hline & & & & \\
\hline & & & & \\
\hline \multicolumn{5}{|l|}{ Individual patient level $(\mathrm{N}=\mathrm{I}, \mathbf{4 5 8})$} \\
\hline Total number of patients who were tested ${ }^{\ddagger}$ & $\mathrm{I}, 233(100)$ & $233(100)$ & $1,000(100)$ & 0.519 \\
\hline HER2- & 97I (78.8) & $178(76.4)$ & $793(79.7)$ & \\
\hline HER2 equivocal & $19(1.9)$ & $4(1.7)$ & $15(1.5)$ & \\
\hline HER2+ & $238(19.3)$ & $51(21.9)$ & $187(18.7)$ & \\
\hline Proportion of HER2+ patients of those with ESBC & $245(100)$ & $58(23.7)$ & $187(76.3)$ & \\
\hline \multicolumn{5}{|l|}{ Test level $(\mathrm{N}=\mathrm{I}, 802)$} \\
\hline $\mathrm{IHC}^{\S}$ & I,359 (75.4) & $242(17.8)$ & $\mathrm{I}, \mathrm{I} \mid \mathrm{7}(82.2)$ & $<0.001$ \\
\hline Score 0 (negative) & $311(22.8)$ & $94(38.8)$ & $217(19.4)$ & \\
\hline Score I+ (negative) & $453(33.3)$ & $43(17.8)$ & $410(36.7)$ & \\
\hline Score $2+$ (equivocal) & $345(25.5)$ & $50(20.7)$ & $295(26.4)$ & \\
\hline Score 3+ (positive) & $232(17)$ & $53(21.9)$ & $179(16)$ & \\
\hline FISH"I & $423(23.47)$ & $66(15.6)$ & $357(84.4)$ & 0.871 \\
\hline Nonamplified & $333(81.6)$ & $56(84.9)$ & $282(79)$ & \\
\hline Equivocal & $15(3.7)$ & $2(3)$ & $13(3.6)$ & \\
\hline Amplified & $56(13.7)$ & $8(12.1)$ & $48(13.5)$ & \\
\hline
\end{tabular}

Notes: *Eleven tests were performed with other types of tests; ${ }^{\dagger} P$-value from the chi-square test of homogeneity; ${ }^{\ddagger}$ unknown final HER 2 result in one tested patient $(0.1 \%)$; \$ $/ \mathrm{HC}$ result unknown/other in eight (0.6\%) tests; "FISH result unknown/other in 19 (4\%) tests. ASCO/CAP guidelines were established in 2007. ${ }^{2}$

Abbreviations: HER2, human epidermal growth factor receptor 2; ASCO/CAP, The American Society of Clinical Oncology and the College of American Pathologists; n, sample number; NCCN, National Comprehensive Cancer Network; N, total number; ESBC, early-stage breast cancer; IHC, immunohistochemistry; FISH, fluorescence in situ hybridization.

considered HER $2+(18.7 \%$ versus $21.9 \%$ [post- versus pre-2007]) following guideline publication.

\section{Discussion}

This is one of the few cancer registry studies assessing the rate of HER 2+ disease and the use of HER2-directed therapy in ESBC. This study also examined HER2 testing patterns, expression, and the use of reflex testing in patients with ESBC, providing new insight into HER2 testing patterns and the degree to which the results of the test influence prescribing patterns at an academic cancer hospital. This study was also able to uniquely assess the reasons for the lack of use of HER2-directed therapy in HER2+ patients. A longitudinal assessment of HER2 test results before and after the publication of the 2007 ASCO/CAP HER2 testing guidelines was also conducted.

In this study, the overall prevalence of HER2 positivity in ESBC patients was determined to be $17 \%$. This result is consistent with other reported estimates in the literature. ${ }^{13-15}$ A study of ESBC patients from the national SEER registry estimated HER 2 positivity at $16 \%$, with more stage 2 and fewer stage 1 patients in the cohort. ${ }^{13}$ An additional study documented the proportion of patients that were positive for PR, ER, and HER2 at $11.4 \%$ compared to our report of $13.3 \%{ }^{14}$

Since 2001, ASCO and the NCCN have recommended HER2 testing for all newly diagnosed, recurrent, or metastatic breast cancers. ${ }^{1,16}$ However, despite these recommendations for universal HER2 testing, our analysis identified 26 (2\%) patients in whom no HER2 status could be confirmed, either by chart review or by review of the documentation in the tumor registry. Similar studies of breast cancer patients have also demonstrated a lack of universal testing. ${ }^{13,15,17}$ Increased institutional efforts are needed to ensure proper documentation of HER2 testing results.

The subgroup analysis of this study is consistent with previous reports. The published literature indicates the different incidence rates of HER 2 positivity by age ${ }^{18}$ Cronin et $\mathrm{a}^{13}$ found rates of HER 2 positivity of $19 \%$ in female ESBC patients $<50$ years of age and $15 \%$ in those $\geq 50$ years of age. A study of invasive breast cancer patients found the highest percentage of HER2 positivity (24.7\%) in the youngest age cohort (20-39 years). ${ }^{19}$ Rates of HER2 positivity have also been shown to differ according to ER and PR status. Studies of breast cancer patients have demonstrated an inverse relationship between HER2 and ER levels. ${ }^{13,19,20}$ Similarly, this study also indicated significant differences in the percentage of HER2 positivity across ER status: the highest percentage of HER2 positivity was found in those with ER- tumors.

This study also assessed the use of reflex testing. The ASCO/CAP and NCCN guidelines for HER2 testing recommend either IHC or FISH as an initial testing method., Utilization of IHC testing with reflexive testing to FISH for IHC2+ results is a common algorithm across many institutions, since IHC testing is less expensive to conduct and can 
be preserved on slides. ${ }^{15,17}$ Testing methods that rely on newer technologies, such as chromogenic in situ hybridization, were used infrequently in this study. Given the availability and benefit of HER2-directed therapies, the need for correct and reproducible HER2 test results are paramount. ${ }^{21}$ Oftentimes, a single reflex test is all that is needed to obtain definitive HER2 status in equivocal cases. ${ }^{4}$ In this study, reflex HER2 FISH testing was able to provide a definitive HER2 result following equivocal IHC results in the majority (269 of 291, or $92.4 \%$ ) of cases, identifying an additional 29 (10\%) HER2+ patients who would benefit from available HER2-directed therapies. As such, reflex testing should be underscored as a potential quality indicator in clinical practice.

The 2007 ASCO/CAP HER2 testing guidelines raised the threshold for a positive IHC assay to $>30 \%$ uniform intense HER2 membrane staining versus the previously defined $>10 \%$ staining. ${ }^{10}$ Our study results indicate that this guideline change may have resulted in reclassifying approximately $5.9 \%$ of $\mathrm{IHC} 3+$ tumors to $\mathrm{IHC} 2+$, which is consistent with other reports of increased $\mathrm{IHC} 2+$ tumors during this time period. ${ }^{22,23}$ Additionally, although not significant, the proportion of HER2+ patients did decrease after guideline publication $(4.2 \%)$, which is consistent with other reports documenting a $3 \%$ difference. ${ }^{23}$ These results suggest that $3 \%-4 \%$ of ESBC patients under the 2007 HER2 testing guidelines were not eligible for adjuvant trastuzumab based on the 2007 ASCO/CAP IHC staining recommendations unless they were reflex tested with FISH.

Recently, the ASCO/CAP guidelines were updated (2013), reverting the 2007 recommendation for circumferential, intense, and complete membrane staining in $>30 \%$ cells for IHC equivocal (IHC2+) and positive (IHC3+) tumors to the previous definition of $>10 \%$, which was used prior to 2007 and was included in the adjuvant trastuzumab trials. ${ }^{10}$ Additionally, the FISH dual-probe HER2/CEP17 (HER2/ chromosome 17 centromere) ratio for HER2 positivity was also reverted to $\geq 2.0$ from $\geq 2.2$, as proposed in the 2007 guidelines.

Overall, use of HER2-directed therapies within the study population appeared appropriate. All of the patients receiving HER2-directed therapies were considered HER2+. Other studies have demonstrated similar findings, with reports of $100 \%$ of trastuzumab utilization in HER2+ patients. ${ }^{15,17}$ However, there are some opportunities for improvement in the utilization of HER2-directed therapies in those patients that tested HER2+, but that did not receive any targeted therapy. This study allowed for the exploration as to why patients were not receiving HER2-directed therapy in HER2+ cases. The most commonly reported reason for not administering HER2-directed therapy was a low risk of recurrence based on stage or other treatments received. The use of HER2-directed therapies for HER2+ subcentimetric $(<5 \mathrm{~mm} ;>5 \mathrm{~mm}$ but $<10 \mathrm{~mm})$ node-negative tumors has not been studied in randomized controlled trials; however, these tumors may have a higher risk of recurrence than HER2tumors. ${ }^{24,25}$ Current NCCN guidelines recommend the use of HER2-directed therapy for subcentimetric HER2+ tumors on a case-by-case basis, balancing the risk of toxicity with the uncertain absolute benefit of therapy. ${ }^{1}$ Further research is needed to assess the benefit of HER2-directed therapy in subcentimetric, node-negative tumors. In eleven of 59 cases, nearly $20 \%$ of the time, there was no explanation given as to why HER2-directed therapy was not administered. In other instances, the reasons for a lack of use appeared justifiable. Overall, utilization of HER2-directed therapies in HER2+ patients, as well as appropriate documentation for the lack of use, should also be considered key quality indicators in $\mathrm{ESBC}$ in addition to reflex testing.

There are several limitations of this research that should be considered. The results from this study were from a single institution and may not be generalizable to a larger population or geographic area. Data from medical charts and tumor registries can be subject to missing data and coding errors. Information bias is possible since the method used to obtain the tissue sample (core biopsy or excised tissue) was not recorded. The source of the tissue (primary tumor versus lymph node or distant metastases) may influence the result of a HER 2 test. ${ }^{26}$ Additionally, this analysis did not assess blockto-block heterogeneity ${ }^{27}$ or chromosome 17 polysomy, ${ }^{28,29}$ which may impact HER2 interpretation. Final HER2 status was determined, when available, from data contained within the patient's medical chart. In instances when the information was not available in the chart, the final HER2 status was determined by the status recorded in the tumor registry. HER2 status was not a recorded measure in the tumor registry until 2010. Beginning in 2010, concordance of HER2 status between the medical charts and tumor registry could be assessed. The time between HER2 tests was not measured as part of this study, but it may be an interesting consideration for future studies. Barron et $\mathrm{al}^{15}$ found a mean delay of 45 days between a patient's first equivocal result and a second HER2 test. The present study did not have a sufficient sample size to detect the impact of 2007 ASCO/CAP HER2 testing guidelines on HER2 test results. Additionally, this study was conducted prior to the implementation of the 2013 ASCO/ CAP guidelines and, therefore, future studies are needed to assess the potential impact of these updated guidelines on HER2 testing outcomes in community settings. 


\section{Conclusion}

This study is one of few studies that linked a cancer registry with an EHR database to assess the rate of HER2+ disease in ESBC. The HER2+ rate was found to be $17 \%$ and within the range of rates reported in previously published studies. The importance of reflex testing should be underscored; a confirmatory test with FISH after an initial test with $\mathrm{IHC} 2+$ helped to identify additional HER $2+$ tumors by approximately $10 \%$. The use of trastuzumab among those with HER2+ tumors was consistent with guideline recommendations. Lastly, the publication of the 2007 ASCO/CAP guidelines on HER2 testing appeared to have an impact on testing practices during prior years, resulting in a shift in the classification of tumors from $\mathrm{IHC} 3+$ to $\mathrm{IHC} 2+$, as well as an increase in reflex testing. Guidelines on HER2 testing are influential and appear to have an impact on testing practices and the identification of patients with HER2+ disease.

\section{Acknowledgment}

The authors would like to acknowledge Reed Barney and Brian Oberg from the University of Utah Enterprise Data Warehouse for data extraction and management.

\section{Disclosure}

This work was supported by an unrestricted research grant by Genentech, Inc. The authors report no other conflicts of interest in this work.

A portion of this work was presented at ASCO's Quality Care Symposium, November 1-2, 2013, Manchester Grand Hyatt, San Diego, CA, USA.

\section{References}

1. National Comprehensive Cancer Network. NCCN Clinical Practice Guidelines in Oncology: Breast Cancer. Fort Washington, PA: National Comprehensive Cancer Network; 2014. Available from: http://www.nccn. org/professionals/physician_gls/pdf/breast.pdf. Accessed June 10, 2014.

2. Wolff AC, Hammond ME, Schwartz JN, et al; American Society of Clinical Oncology/College of American Pathologists. American Society of Clinical Oncology/College of American Pathologists guideline recommendations for human epidermal growth factor receptor 2 testing in breast cancer. Arch Pathol Lab Med. 2007;131(1):18-43.

3. College of American Pathologists. ASCO-CAP HER2 Test Guideline Recommendations: Summary of Guideline 2007 and 2013 Recommendations. Northfield, IL: College of American Pathologists; 2013. Available from: http://www.cap.org/apps/docs/committees/immunohistochemistry/ summary_of_recommendations.pdf. Accessed September 18, 2014.

4. Clay MR, Iberri DJ, Bangs CD, Cherry A, Jensen KC. Clinicopathologic characteristics of HER2 FISH-ambiguous breast cancer at a single institution. Am J Surg Pathol. 2013;37(1):120-127.

5. Moja L, Tagliabue L, Balduzzi S, et al. Trastuzumab containing regimens for early breast cancer. Cochrane Database Syst Rev. 2012;4: CD006243.

6. Mitri Z, Constantine T, O'Regan R. The HER2 receptor in breast cancer: pathophysiology, clinical use, and new advances in therapy. Chemother Res Pract. 2012;2012:743193.
7. Dekker TJ, Borg ST, Hooijer GK, et al. Determining sensitivity and specificity of HER2 testing in breast cancer using a tissue micro-array approach. Breast Cancer Res. 2012;14(3):R93.

8. Gown AM. Current issues in ER and HER2 testing by ICH in breast cancer. Mod Pathol. 2008;21 Suppl 2:S8-S15.

9. Wolff AC, Hammond ME, Hicks DG, et al; American Society of Clinical Oncology; College of American Pathologists. Recommendations for human epidermal growth factor receptor 2 testing in breast cancer: American Society of Clinical Oncology/College of American Pathologists clinical practice guideline update. Arch Pathol Lab Med. 2014;138(2):241-256.

10. Wolff AC, Hammond ME, Hicks DG, et al; American Society of Clinical Oncology; College of American Pathologists. Recommendations for human epidermal growth factor receptor 2 testing in breast cancer: American Society of Clinical Oncology/College of American Pathologists clinical practice guideline update. J Clin Oncol. 2013;31(31): 3997-4013.

11. Wolff AC, Hammond ME, Schwartz JN, et al. American Society of Clinical Oncology/College of American Pathologists guideline recommendations for human epidermal growth factor receptor 2 testing in breast cancer. J Clin Oncol. 2007;25(1):118-145.

12. Charlson ME, Pompei P, Ales KL, MacKenzie CR. A new method of classifying prognostic comorbidity in longitudinal studies: development and validation. J Chronic Dis. 1987;40(5):373-383.

13. Cronin KA, Harlan LC, Dodd KW, Abrams JS, Ballard-Barbash R. Population-based estimate of the prevalence of HER-2 positive breast cancer tumors for early stage patients in the US. Cancer Invest. 2010;28(9):963-968.

14. Parise CA, Bauer KR, Brown MM, Caggiano V. Breast cancer subtypes as defined by the estrogen receptor (ER), progesterone receptor (PR), and the human epidermal growth factor receptor 2 (HER2) among women with invasive breast cancer in California, 1999-2004. Breast J. 2009;15(6):593-602.

15. Barron JJ, Cziraky MJ, Weisman T, Hicks DG. HER2 testing and subsequent trastuzumab treatment for breast cancer in a managed care environment. Oncologist. 2009;14(8):760-768.

16. Bast RC Jr, Ravdin P, Hayes DF, et al; American Society of Clinical Oncology Tumor Markers Expert Panel. 2000 update of recommendations for the use of tumor markers in breast and colorectal cancer: clinical practice guidelines of the American Society of Clinical Oncology. J Clin Oncol. 2001;19(6):1865-1878.

17. Goddard KA, Bowles EJ, Feigelson HS, et al. Utilization of HER2 genetic testing in a multi-institutional observational study. Am J Manag Care. 2012;18(11):704-712.

18. Parise CA, Bauer KR, Caggiano V. Variation in breast cancer subtypes with age and race/ethnicity. Crit Rev Oncol Hematol. 2010;76(1): $44-52$.

19. Fehrenbacher L, Habel LA, Capra AM, Anthony A, Li X. Incidence, demographic and tumor characteristics of HER2-positive invasive breast cancer in a large, unselected population, 2000-2006. Cancer Research. 2009;69(24): Supplement 3

20. Konecny G, Pauletti G, Pegram M, et al. Quantitative association between HER-2/neu and steroid hormone receptors in hormone receptor-positive primary breast cancer. J Natl Cancer Inst. 2003;95(2):142-153.

21. Viale G. Controversies in testing for HER2. American Society of Clinical Oncology 2011 Educational Book. 47th Annual Meeting; June 3-7, 2011; Chicago, IL. Alexandria, VA: American Society of Clinical Oncology; 2011.

22. Schalper KA, Kumar S, Hui P, Rimm DL, Gershkovich P. A retrospective population-based comparison of HER2 immunohistochemistry and fluorescence in situ hybridization in breast carcinomas: impact of 2007 American Society of Clinical Oncology/College of American Pathologists criteria. Arch Pathol Lab Med. 2014;138(2):213-219.

23. Shah SS, Ketterling RP, Goetz MP, et al. Impact of American Society of Clinical Oncology/College of American Pathologists guideline recommendations on HER2 interpretation in breast cancer. Hum Pathol. 2010;41(1):103-106. 
24. Curigliano G, Viale G, Bagnardi V, et al. Clinical relevance of HER2 overexpression/amplification in patients with small tumor size and node-negative breast cancer. J Clin Oncol. 2009;27(34):5693-5699.

25. Gonzalez-Angulo AM, Litton JK, Broglio KR, et al. High risk of recurrence for patients with breast cancer who have human epidermal growth factor receptor 2-positive, node-negative tumors $1 \mathrm{~cm}$ or smaller. J Clin Oncol. 2009;27(34):5700-5706.

26. Carlson RW, Moench SJ, Hammond ME, et al; NCCN HER2 Testing in Breast Cancer Task Force. HER 2 testing in breast cancer: NCCN Task Force report and recommendations. J Natl Compr Canc Netw. 2006;4 Suppl 3:S1-S22; quiz S23-S24.
27. Ohlschlegel C, Zahel K, Kradolfer D, Hell M, Jochum W. HER2 genetic heterogeneity in breast carcinoma. J Clin Pathol. 2011;64(12): 1112-1116.

28. Vanden Bempt I, Van Loo P, Drijkoningen M, et al. Polysomy 17 in breast cancer: clinicopathologic significance and impact on HER-2 testing. J Clin Oncol. 2008;26(30):4869-4874.

29. Vranic S, Teruya B, Repertinger S, Ulmer P, Hagenkord J, Gatalica Z. Assessment of HER2 gene status in breast carcinomas with polysomy of chromosome 17. Cancer. 2011;117(1):48-53.

\section{Publish your work in this journal}

Breast Cancer: Targets and Therapy is an international, peerreviewed open access journal focusing on breast cancer research, identification of therapeutic targets and the optimal use of preventative and integrated treatment interventions to achieve improved outcomes, enhanced survival and quality of life for the cancer patient.
View the full aims and scopes of this journal here. The manuscript management system is completely online and includes a very quick and fair peer-review system, which is all easy to use. Visit http:// www.dovepress.com/testimonials.php to read real quotes from published authors.

Submit your manuscript here: http://www.dovepress.com/breast-cancer---targets-and-therapy-journal 